\title{
Téoros
}

Revue de recherche en tourisme

\section{Bonjour ma ville}

\section{Bernard Noulin}

Volume 10, numéro 3, novembre 1991

Destination Montréal : d'hier à demain

URI : https://id.erudit.org/iderudit/1079207ar

DOI : https://doi.org/10.7202/1079207ar

Aller au sommaire du numéro

\section{Éditeur(s)}

Université du Québec à Montréal

\section{ISSN}

0712-8657 (imprimé)

1923-2705 (numérique)

Découvrir la revue

\section{Citer ce document}

Noulin, B. (1991). Bonjour ma ville. Téoros, 10(3), 60-61.

https://doi.org/10.7202/1079207ar d'utilisation que vous pouvez consulter en ligne.

https://apropos.erudit.org/fr/usagers/politique-dutilisation/ 
l'exterieur; la ne ou il hobite, l'ecole, l'arena. le cafe quill frequentait de mentre que les prindpolesentreprisesquiconaderisent l'activite economique decenteportie de laville Liseléne, de son cole, fille dimmigres suisses, ayon realise une efude sur le rourisme d Montreal, miaemmené le long du Sanr-Lourenr d'Est en Quest jusqu'ou Cop 5i-Jocques. Cerait la premiere fois, depuis 14 ans! Cela mia fortement impressionne de decouvir ce liftoral que j'ignorais parce qu'd Bruxelles, nous sommes frumbs de la disporition de notre riviere - la Senne - dons une canalisarion sourerraine de meme que les divers bros de conouxpenerront ou centre de la ville, en patte d'oie, comme d Amsterdom, er rembloye depuis 1905 !! Pourquol les Montrélais que je connais ne miont jomais fait porcourir avec le même enthousiosmedpied, avelo ouenvoifure les berges du fleuve er reveler la beoure er la diversire des maisons er propriétes résidentielles? Les en jeux soooux er economiques? Les menoces ecologiques?

\section{Conclusion}

En definirive, je me rends compte à quel poinjiol ete un privilégie dons certe decou verte de Montreal er de ses hobitonts. J'al profire des reseoux des relations de Rithard er Louis dons lesquels ils miont iniroduir. Com ment les autres tourisres dogrement, de conferences ou d'affaires, selon leurs centres d'inverers, pourcient-ils beneficier des mentres dhonces de renconires er de decouvertes?

Seul un lieu, reperable er diffuse, anime par des personnes ressources du milieu ossociatif, pourrait leur liver les listes d'adresses, les cartes de leurs localisations, les calendriers de leurs octivités ouvertes oux visileurs - fêres, exposirions, visires thematiques guidees-concernont les organisations socioculfurelles provinciales, erthniques er locales, siruees a Montrél? Les odresses er horcires des videorheques, ou lion peur revoir les rémolgnages des evenements qui ont marque son hisioire ou les documenraires qui livent des clefs dinterpretarion des diverses realites el fonctions? Pourquoi les services de presses el de relations publiques de l'Universire, des ministeres provinoloux de Rodio Conoda, de la Ville de Monmeal... riont-ils pos des expositions permonentes presentant leur histoire er leurs projets occompognees de vidéos ou d'exposilions remporaires er themariques, sur des aspects particuliers propres d Montreal? Enfin, ne foudrair-il pas un Cenire dinterpretationsurles relations systemiques du fleuve Saint Lourent er de la Ville de Montreol comme il śen est ouvert un sur lo Loire ou le Cenrre de la Mer a Doulogne, en France? J'oppelle de rous mes voux lo cearion de ces initiatives goges dun tourisme culturel de rencontres er de patages entre les Montrécalais euxmemes, les Quebecois er Conodiens ainsi que les visiteurs etrangers!

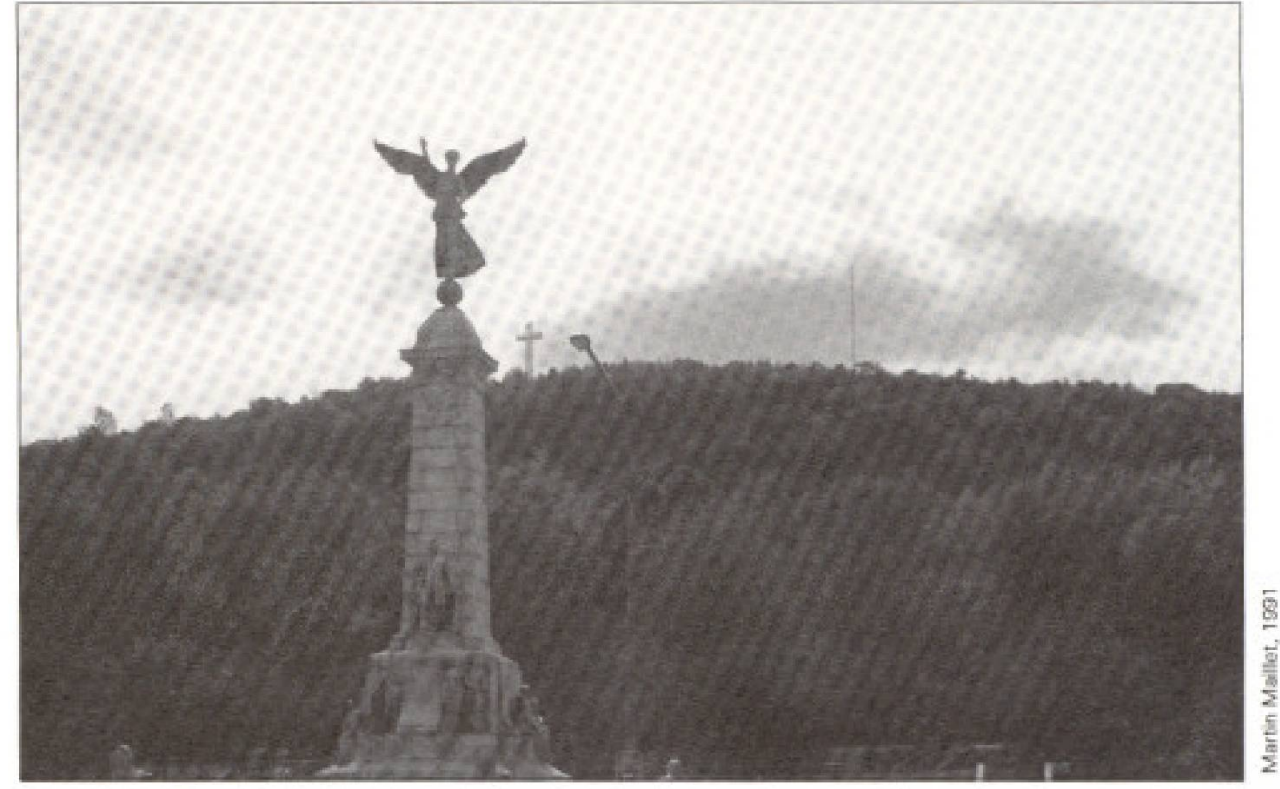

Bonjour ma ville

\section{par Bernard Noulin}

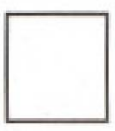

Donjour mo ville - re voild d nouve au plus belle que jamois sous le soleil de cene fin d'ere. Avec lo chaleur, Montreal porfois l"ere tu deviens africaine er, meme si Cartier nía pos voyoge a l'envers de l'hiver, la rue Peel, l'ete. Cest un peu de Modrid ou de Rome a certe epoque.

Pour moi depuis 15 ans, tour commence de la même foçon. Apres Terre-Neuve, deja les locs de la belle province me font signe, deja je sois quiou bour, lorsque lo machine survolero l'outoroure des Laurentides, il y aura Mirabel. J'aime ef je déteste Mirabel. J'aime Mirabel (ron ther ellephont blonc) lorque, dons l'ere roride ou dons l'hiver glacial, simmobilise le monstre. Cest roujours un parcours iniriorique, je nesuis chez moi que lorsque sedecoupe, sur le ciel, ton centreville, la montagne ef I'Orotoire. Decoupoge sublime qui foil que. pour quelques instonts, ru deviens theórre d'ombres, Je dereste Mirabel lessoirs de retour. Mais en cer instont, je ne veux pos en porler.

Souverir mes amis dial ou de labbos (mois ou est id ou est lobas?) me demandent pourquo je t'aime. Pourqual, loin des rives de la Seine. je te irouvesuperbe, toi qui ríapos de Tuileries, de Loume ou de Pontheon.

A vai dire, ru as beoucoup plus. Bien sôr que ru n'os pos ce qui fair le charme de ma ville narole (que joime presque ourani) mois ru as pour moi des imoges fugoces er vives qui, depuis 15 ans, ont risse le fil de ma passion.
Comme un marin doutomne, lorque deja le vent descendu du Labrodor cherche o rengourdir. Tu oscilles olors entre le soleil qu Irve son dernier combar er le froid qui wa re soisir juqu'ou printemps. A cette epoque, tes deux sont superbes, souvent rouges, l'air lim. pide. A ces rouges celestes se melent ceux de la montogne, teintes superbes, melonge de feucomrne pourbrulerune derniêre fols l'eclor de ronérequise meure Cemarinta, llyodejo bien des soisons, j'érais dans un hotel du cenrreville donnani sur le fleuve er le pont Jocques Cartier. Cérair 11 y a longremps er pourtant il reste en ma mémoire. Dentelle de fer se dérachant sur ce ciel rouge. Dons le silence de certe chambre, je voyais le rafic posser le fleuve, ron fleuve er auroni de sujets allont dans la dié, allon vers Derri ou Laurier. ville avalont comme un colosse, ville se nou rissant de ceux de Longueuil ou St-Jean.

Cerair, pelle mêle, un qurre jour sur Coredes. Neiges, un jour d'éte el une pluie borrone thoude, omendue depuis lemorin pour souloger lescorps, les ortres des moux queru infliges $p$ ar res choleurs ront souhailees au coeur de jonvier mais rellemenr erouffanres que porfois res hobironis disent que l'hiver a du bon.

Je ne sais pourquoi celte image est en moi. II pleuvair wo boire debout ${ }_{r}$ les rares passonts pressaient le pos, il pleuvait er, jetais la seul regardant la montagne érincellanre sochoni quid trovers le rideou de plute, mes dhers ecureuilsretrouvilent pouruntemps seulement la plenirude des themins, sentiers er bonos. Jerois ló, la montogne érair belle, lustree, brillante, comme une ve imoginaire offerted ceux qui soven quel remain d'oventure elle codne.

Cérair oussi un dimanche soir en novembre Depuis le morin nous le sovions. Ton ciel erair 


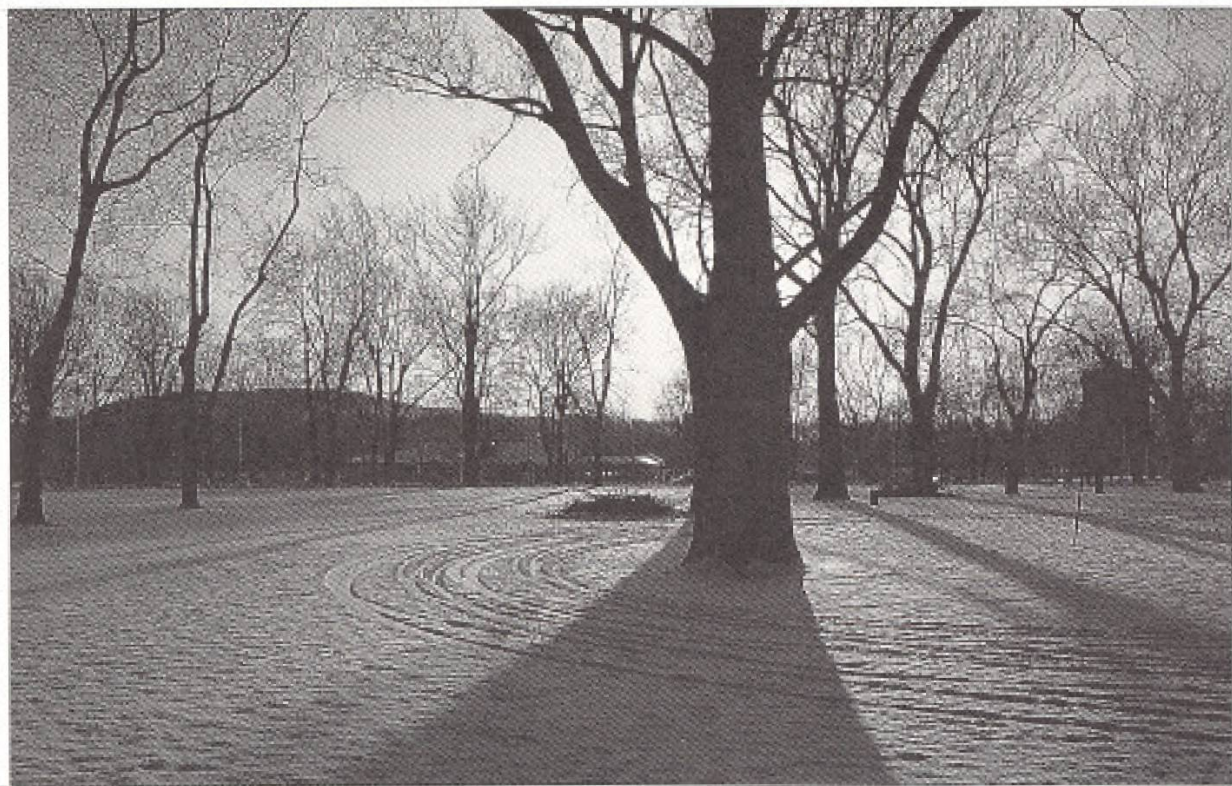

gris, ovec cerre douceur de l'air de la fin d'outomne (non pos douceur, frotheur serait le mot mais une fraicheur envoorante). Dejó les bruits se foisaient plus discrets. Ton ciel gris n'eraltposmenocant, mois nous sovions qu'elle erait la taple. Nous la guetrions, nous l'attendions. Et puis wers certe fin d'opres-midi, elle est venue, liquide et molle tombant du del. Elle s'installair portout prenant possession de son remircire de rol. Vicrorieuse de la ville. Montred, elle r'offre le negarif d'une phoro eremelle, eller'offre le spectodle unique d'une meneuse de revue descendant l'escalier des degres centigrodes.

Certe vedette emponodiee de blonc, elle est romaîtresseexigeante, rentooulaire. Er ce soirla, dans lesilence de tesrues dans lesilence de ma nue, le bruir mär venant de la ruelle, celui d'enfonts jouant seuls ou hockeycomme pour prouver que la vie, to vie, mo vile, es la plus forre

Un mor sur res ruelles ef res balcons (Oh! pardon, galeries). Tu es, mo ville, comme Jonus a deux faces. Tu montres res allees, res ovenues, tes galeries, ensembles disporares mais qui s'harmonisent avec tes maisons. Entrectoisement, dedale descoliers, de balus rrodes, de fleurs et de bicyclertes, l'éré el gloce (arention danger), l'hiver.

Deux foces tu as, car derriere res decors comme ou thétre, il y a une ourre vie, celle de la ruelle. Tes habitants wont sur la grande scene de lo vie, ils entrent, sortent mais sont euxmenernes core cour.

Joime ces ruelles hiver comme êré, je sais que u ru deviens roi-mème. Souvent ou hasard de mes erronces, je me retrouve au Plateou. Rue Pochel (er dons lo ruelle), je cherche encore
Morcel el son that; l'ere je guerre la grosse dameerson interminoble voyage sur sachaise a bascule. Des fois aussi, je therche, ou Parc Lafontaine, les rires oux edors de la rencontre de Gercrder de Therese. Aurant d'images, de souvenirs que je dois a l'un de tes chantres. images qui font que, venu de mon île de France, j'ai un peu d'enfonce ici.

Mais je t'aime surtout pour ton hamonie. C'erail en mai, jovais besoin de toi, de te posseder, j"ovois laisse Paris pour venir woir tes premiers rayons de soleil. J'avais besoln de rai comme toujours. Comme une drogue. Dedoignont ton mérro et ses mysterleux messog. es $3377 n$ communiquez, $\times 212 *$ communiquez, je pense en entendant cela d̀ une machine infernale devoreuse de chair. J'avais entrepris, je ne sais pourquol, de rejoindre le belvedere du mont Royal par cer escolier interminable qui commence au bour de Peel (je cois) Cerair vaiment les premiers jours du printemps, ici ou lä, quelques ploques blandhes arrestoient que l'hiver riercir pas loin. Le soleil poinrair encore pâle, tiéde solell qui donne la vie qui explosera o la Si-Jean Doptiste ou th devens bleve er blanche, fleur de lys dans son ecrin, comme pour montrer woux outres. que ru es lo belle Fronce. Je montois er o mithemin, me suis ossis pour r'odmirer. J'aime tes immeubles, equilibre raisonnable, sans le giganisme clinquant de Toronto ou de Philadelphie.

Je crois que ce qui foir ce thorme unique, cest que depuis lo montogne (le mont Royal), on peut presque toucher res grofte-ciels tellement ils sont pres. De la montogne, on les domine. L'homme les surposse, il riest pos ou millieu perit, insignifioni, il es ou-dessus, maitre de lo marière er de l'espoce. Tu es la seule ville de ce continent d offrir ce lien entre ce que la noture a fait er ce que l'homme a realise.
J'etais donc lá ossis sur ce banc re regardant r'odmirant quand, a coré de moi, est venul'un de reshobitants, l'ecureuil, fouineuret curieux Lui comme inoi te regordon, humont le prin. remps: lequel de nous érair le plus eroné, je ne le sais pos encore? Mais il me reste le souvenir d'une parcelle de plénirude, assis cored-cole, nous riavons aime longremps.

Je ne peux re dire tout ma dhereville, tous ces souvenirsque, depuis presque 15 ans, je ploce ou grenier de mo memoire pour plus rord.

II mien reste un d te confier, mais ó toi seule ment. Un soir, une bolte d dhansons, fard, tres rord, les rouristes sont loin, ils onr regogné leur hôrel. II reste là ceuxpour qui ici la nuir es une fere, laissont alors le rempser labiere emplir les esprits, ils ont chante. Ils ferraient la salle, les musiciens, ils feroient ceux du Quebec. Ce jour-lo moville, certe nuit $\mathrm{t}$, joi su quel'on ne peur aborrre un peuple qui chante. Certe nuitlá. j'ai su que ru resteras toujours la premiere cité francophone endehors du veux poys français.

Cest rout, ma ville. Lorsqu'd Paris ou a Mon meal, on me demande pourquoi je r'aime, je ne peux pos dire tour celo ef je reprends Cocteau qui disair: *la poesle c'esi comme les occidents de chemin de fer, ça ne s'explique pos, ça se ressent"

Lorsque je remouve lo roure de Mrabel, je sois l'endroir ou, me reroumant, je wois encare t'opercevoir, en mombres, en doir obsour. Je me retoume, je te mumure: "Solur mo ville* et parfois, deroumant mon regard, je re pleure.

Fasse que jamals je ne sache que ciest lo derniere fois, que roujours me reste, thevillee ou fond du ventre, la certirude quil y en quro encore une. Une seule pour re dire: Nontreal, je t'aime. 